\title{
Canais Iônicos na Epilepsia
}

\section{Ion Channels in Epilepsy}

\author{
Alysson S. da Silva ${ }^{1}$, lara L. L. de Souza², Bagnólia A. da Silva ${ }^{3}$
}

\begin{abstract}
RESUMO
Dados da Organização Mundial da Saúde apontaram uma prevalência mundial de epilepsia por volta de 50 milhões de pessoas. A crise epiléptica é um período de excitação síncrona anormal de uma população de neurônios. O balanço entre sistemas de controle que impedem uma descarga de potencial de ação excessiva nos neurônios e outros sistemas que facilitam a excitação mantém o bom funcionamento do sistema nervoso. Este trabalho teve como objetivo compilar informações sobre o envolvimento das canalopatias no processo fisiopatológico das crises epilépticas. Foi realizada uma revisão de literatura, através de pesquisas bibliográficas utilizando os bancos de dados Medline/PubMed, Scielo, Scirus, Wiley Online Library e Science Direct. Para tal finalidade, os descritores utilizados foram: epilepsia, crises epilépticas, canais iônicos, canalopatias e epidemiologia, em idiomas português e inglês. As investigações genéticas fizeram com que algumas síndromes epilépticas fossem consideradas canalopatias e, por conseguinte, aumentaram o entendimento da fisiopatologia dos transtonos convulsivos. Dada à incapacidade de cura dos fármacos disponíveis atualmente, o grande desafio para as pesquisas científicas é a descoberta de um tratamento capaz de prevenir ou reverter a epilepsia.
\end{abstract}

Palavras-chave. Crise Epiléptica, Canais lônicos, Canalopatias.

\section{Introdução}

Hipócrates, por volta dos anos 400 a. C. , reconheceu a epilepsia como uma disfunção cerebral, indo contra o conceito da época de que as crises eram maldições dos deuses. Ele escreveu o primeiro livro sobre epilepsia, "Sobre a Doença Sagrada", uma das obras mais importantes do Corpus hippocraticum. Durante séculos, possessão, magia e bruxaria foram associadas à epilepsia. Em 1494 foi lançado o Malleus Maleficarum, um manual de caça às bruxas, onde as crises serviam como características para identificar as feiticeiras ${ }^{1}$. Até hoje, a etiologia das epilepsias ainda não é totalmente compreendida e as pesquisas na área são constantes.

Dados da Organização Mundial da Saúde (OMS) apontam uma prevalência mundial da epilepsia por volta de 50 milhões $(0,4-1 \%)$ de pessoas, onde aproximadamente $90 \%$ delas vivem em países em desenvolvimento. A taxa de incidência anual nos países desenvolvidos varia de 40-70/100. 000 habitantes, enquanto que nas regiões em desenvolvimento esse valor pode dobrar ${ }^{2}$.

A Associação Brasileira de Epilepsia $(\mathrm{ABE})^{3}$, de acordo com a Liga Internacional Contra a Epilepsia $\left(\right.$ ILAE) ${ }^{4}$, define epilepsia como distúrbio cerebral
1. Farmacêutico, Universidade Federal da Paraíba (UFPB)

2. Farmacêutica, mestranda do Programa de Pós-graduação em Produtos Naturais e Sintéticos Bioativos da UFPB

3. Doutora em Biologia Molecular. Professora do Departamento de Ciências Farmacêuticas e do Programa de Pós-graduação em Produtos Naturais e Sintéticos Bioativos da UFPB
CORRESPONDÊNCIA: Bagnólia Araújo da Silva

Universidade Federal da Paraíba (Campus I) CEP 58.051-970, João Pessoa-PB, Brasil

Artigo recebido em 25/11/2013 Aprovado para publicação em 22/04/2014 
causado por predisposição persistente do cérebro a gerar crises epilépticas e pelas consequências neurobiológicas, cognitivas, psicossociais e sociais da condição, caracterizada pela ocorrência de pelo menos uma crise epiléptica.

A crise epiléptica (CE) é um período de excitação síncrona anormal de uma população de neurônios, de caráter interativo e autolimitado. O balanço entre sistemas de controle que impedem uma descarga de potencial de ação excessiva nos neurônios e outros sistemas que facilitam a excitação mantém o bom funcionamento do sistema nervoso. Mecanismos que perturbam o sistema de inibição ou promovem a excitação podem levar ao surgimento das crises ${ }^{5}$. Canais iônicos comandam o processo de excitabilidade dos neurônios, além da comunicação entre eles, através da liberação de neurotransmissores. Logo, não é surpresa que mutações em genes que codificam esses canais e suas subunidades acessórias tenham um papel importante em várias desordens que se associam à hiper ou hipoexcitabilidade neuronal ${ }^{6}$, incluindo as CEs. Esta revisão tem como objetivo reunir informações sobre o envolvimento das canalopatias no processo fisiopatológico das $\mathrm{CE}$.

\section{Método}

Realizou-se uma revisão bibliográfica de estudos da epilepsia nas áreas da Neurologia e Farmacologia. As informações foram retiradas de artigos científicos publicados nos últimos 12 anos, pesquisados nos bancos de dados Medline/PubMed, Scielo, Scirus, Wiley Online Library e Science Direct.

Os descritores utilizados para a busca dos artigos foram: epilepsia, crises epilépticas, canais iônicos, canalopatias e epidemiologia, e suas respectivas traduções para o Inglês.

\section{Resultados}

Canalopatias são doenças adquiridas ou hereditárias resultantes de uma atividade defeituosa de um canal iônico específico. As desordens hereditárias são causadas por mutações em genes que codificam essas moléculas ${ }^{7}$, tanto os canais sensíveis à voltagem $\left(\mathrm{Na}^{+}\right.$, $\mathrm{K}^{+}, \mathrm{Ca}^{2+} \mathrm{e} \mathrm{Cl}^{-}$), quanto os sensíveis aos ligantes, como o receptor nicotínico da acetilcolina (nAChR), receptor GABA do ácido gama-amino-butírico (GABA) e os receptores dos tipos AMPA e NMDA do glutamato. A relação entre os fatores genéticos e o fenótipo da doença determina a classificação das epilepsias em monogênicas, quando a mutação em um único locus gênico é responsável pelo estabelecimento da desordem; e em poligênicas, quando o componente genético apenas determina um limiar de susceptibilidade ${ }^{8}$.

As investigações genéticas, não só farão com que algumas síndromes epilépticas sejam consideradas canalopatias, aumentando o entendimento da fisiopatologia dos transtonos convulsivos, mas também poderão determinar novos alvos para a farmacoterapia ${ }^{9}$.

\section{Canais de sódio sensíveis a voltagem (Na-v)}

\section{$\mathrm{Na}_{\mathrm{v}} 1.1$ (gene SCN1A - cromossomo 2q24.3)}

Mais de 700 mutações no $\mathrm{Na}_{\mathrm{v}} 1.1$ tem sido relacionadas às síndromes epilépticas. Aproximadamente trinta mutações missense, que definem-se pela codificação de um aminoácido diferente do normal (substituição de um nucleotídeo em um determinado códon), foram reportadas em famílias com Epilepsia Generalizada com Crises Febris Plus (GEFS+) ${ }^{10}$. Essa síndrome é uma epilepsia mais leve em que a criança apresenta crises febris, evoluindo para epilepsia generalizada na fase adulta. ${ }^{11}$

Lossin et al. ${ }^{12}$ estudaram os efeitos de três mutações nos resíduos de aminoácidos T875M, W1204R e R1648H em células não neuronais e a anormalidade funcional comum observada foi uma inativação desse canal, levando ao aumento da corrente persistente de $\mathrm{Na}^{+}$. Esta corrente persistente diminui o limiar para o disparo de potenciais de ação, o que resulta diretamente em hiperexcitabilidade.

Outra síndrome relacionada com mutações no $\mathrm{Na}_{\mathrm{v}} 1.1$ é a Epilepsia Mioclônica Severa do Lactente (SMEI) ou Síndrome de Dravet (DS). Mais de 600 mutações têm sido associadas a essa síndrome, onde mais da metade são mutações nonsense ou frameshift e o restante são missense ${ }^{13}$. SMEI é caracterizada por um início precoce, geralmente no primeiro ano de vida, com crises muitas vezes associadas à alta temperatura corporal, podendo evoluir para um estado de mal epiléptico e deterioração mental. ${ }^{14}$

Nas mutações nonsense, um determinado códon de um resíduo de aminoácido é substituído por um códon de terminação. Na mutação frameshift, há inserção ou deleção de pares de nucleotídeos (em número não múltiplo de 3). Ambas as mutações geram proteínas truncadas. ${ }^{13}$ 


\section{$\mathrm{Na}_{\mathrm{v}} 1.2$ (gene SCN2A - cromossomo 2q24.3)}

A Epilepsia Familiar Benigna do Neonato/ Lactente (BFNIS) tem início entre dois dias e três meses após o nascimento e é causada por mutações missense no gene SCN2A, que codifica o canal $\mathrm{Na}_{\mathrm{v}}$ 1.2. Scalmani et al. ${ }^{15}$ estudaram o efeito funcional de quatro mutações em neurônios neocorticais transfectados em culturas primárias. A mutação L1330F substituiu um resíduo de leucina por fenilalanina na alça citoplasmática entre o $\mathrm{S} 4$ (sensor) e S5 do DIII e causou um desvio positivo na inativação sensível à voltagem; a mutação L1563V introduziu um resíduo de valina no S2 do DIV do canal e causou um desvio negativo na ativação sensível à voltagem. R223Q e R1319Q substituíram um resíduo de arginina do S4 do DI e DIII respectivamente, aumentando a amplitude da corrente de $\mathrm{Na}^{+}$. Todos os efeitos são consistentes com a hiperexcitabilidade neuronal.

Kamiya et al. ${ }^{16}$ descreveram uma mutação nonsense em SNC2A em um paciente com uma epilepsia de difícil controle semelhante à SMEI. A mutação resultou em um códon de terminação logo após o resíduo Arg-102 (R102X), localizado na porção $\mathrm{N}$-terminal do $\mathrm{Na}_{\mathrm{v}} 1.2$, obtendo-se uma proteína nãofuncional truncada que termina antes da ocorrência do primeiro segmento transmembrana do DI. Os efeitos dessa mutação foram avaliados na linhagem celular HEK293 expressando tanto os canais $\mathrm{Na}_{\mathrm{v}} 1.2 \mathrm{Wt}$ quanto o canal truncado $\mathrm{Na}_{\mathrm{v}} 1.2 \mathrm{R} 102 \mathrm{X}$. Os achados eletrofisiológicos sugeriram que a proteína $\mathrm{Na}_{\mathrm{v}} 1.2 \mathrm{R} 102 \mathrm{X}$ exerce um efeito negativo dominante nos canais $\mathrm{Na}_{\mathrm{v}} 1.2$ normais levando ao quadro epiléptico do paciente.

\section{$\mathrm{Na}_{\mathrm{v}} 1.3$ (gene SCN3A - cromossomo 2q24.3)}

Apenas uma mutação foi descrita no gene SCN3A (K354Q) e foi descoberta em uma criança com uma epilepsia focal resistente aos fármacos anticonvulsivantes. ${ }^{17}$ A mutação K354Q, que substitui um resíduo de lisina na alça que liga S5 e S6 do DI, foi estudada por Estacion et al. ${ }^{18}$ em neurônios hipocampais transfectados. Os resultados mostraram que houve aumento da corrente de $\mathrm{Na}^{+}$, diminuição do limiar de ativação, e disparos espontâneos semelhantes a um desvio despolarizante paroxístico (DDP), fornecendo uma base para o processo de patogenicidade do primeiro caso de epilepsia ligada à mutação no canal $\mathrm{Na}_{\mathrm{v}} 1.3$.

\section{$\mathrm{Na}_{\mathrm{v}} \beta 1$ (gene SCN1B - cromossomo 19q13.11)}

GEFS+ também está associada à uma mutação C121W no domínio Ig extracelular da subunidade $\beta$ auxiliar dos canais de $\mathrm{Na}^{+}$sensíveis à voltagem. Utilizando linhagens celulares de mamíferos, Meadows et al. ${ }^{19}$ observaram que a mutação missense $\mathrm{C} 121 \mathrm{~W}$ gerou uma diminuição da modulação da subunidade $\alpha$ pela $\beta$, levando a um aumento na atividade do canal nos potenciais de membrana hiperpolarizados, em comparação com células que expressam a subunidade $\beta$ normal. A mutação ainda dificultou a interação com moléculas de adesão, evento crítico para a localização do canal. O comprometimento da inativação da subunidade $\alpha$ é o mecanismo que relaciona mutações no SCN1B com a hiperexcitabilidade neuronal na epilepsia. Outras mutações (R85C e R85H) nessa subunidade já foram descobertas, além da relação de pacientes com Epilepsia do Lobo Temporal (TLE) com a mutação $\mathrm{C} 121 \mathrm{~W}^{20}$.

\section{Canais de potássio}

\section{Canais M (genes KCNQ2 e KCNQ3 - cromos- somos 20q13.3 e 8q24. 22)}

Genes KCNQ encodam cinco subunidades $\mathrm{K}_{\mathrm{v}} 7$ $\left(\mathrm{K}_{\mathrm{v}}\right.$ 7. 1- $\left.\mathrm{K}_{\mathrm{v}} 7.5\right)$, onde quatro delas $\left(\mathrm{K}_{\mathrm{v}}\right.$ 7.2- $\left.\mathrm{K}_{\mathrm{v}} 7.5\right)$ são expressas no SNC. Subunidades $\mathrm{K}_{\mathrm{v}} 7.2$ (KCNQ2) e $\mathrm{K}_{\mathrm{v}} 7.3$ (KCNQ3) formam um heterotetrâmero conhecido como canal $\mathrm{M}$, um tipo de canal de $\mathrm{K}^{+}$sensível à voltagem de abertura e fechamento muito lentos. São inativados por receptores acoplados a proteína $\mathrm{G}_{\mathrm{q} / 11}$, tal como receptores muscarínicos da acetilcolina $\mathrm{M}_{1}$ $\mathrm{e}_{3}{ }^{21}$, daí o nome do canal. Canais $\mathrm{M}$ repolarizam a membrana da célula nervosa impedindo disparos repetitivos de potenciais de ação, bem como suprimem a geração espontânea destes.

Mutações nos genes KCNQ2 e KCNQ3 são a causa da Epilepsia Familiar Benigna Neonatal (BFNC), caracterizada pelo surgimento de crises generalizadas ou focais nos primeiros dias de vida e com remissão espontânea em um mês ${ }^{22}$.

\section{Proteína Lgi1 (gene LGl1 - cromossomo 10q23.33)}

A proteína 1 rica em leucina inativada em glioma (Lgi1), produto do gene de mesmo nome é expressa principalmente no cérebro, consiste em um polipeptídio formado por um peptídeo sinal $\mathrm{N}$-termi- 
nal e dois domínios estruturais distintos: um domínio formado por três regiões ricas em resíduos de leucina (LRR) ladeadas por conjuntos conservados de resíduos de cisteína, e o outro domínio formado por sete regiões formadas por repetições de 50 aminoácidos (EPTP ou EAR). Possui duas isoformas, Lgi1 de cadeia longa e de cadeia curta, esta última possuindo apenas uma região EPTP. ${ }^{23}$

Schulte et al. ${ }^{24}$ mostraram que a proteína Lgi1 está associada ao canal $\mathrm{K}_{\mathrm{v}} 1.1$, junto ao canal $\mathrm{K}_{\mathrm{v}} 1.4 \mathrm{e}$ subunidade auxiliar $\mathrm{K}_{\mathrm{v}} \beta 1$, formando o complexo do canal de $\mathrm{K}^{+}$do tipo A nos terminais axônicos; e que Lgi1 seletivamente previne a inibição do $\mathrm{K}_{\mathrm{v}} 1.1$ pela subunidade $\mathrm{K}_{\mathrm{v}} \beta 1$. Esses pesquisadores concluíram que mutações no gene LGI1 tornaram a proteína incapaz de inibir a atividade da $\mathrm{K}_{\mathrm{v}} \beta 1$, o que resultou em canais com uma cinética de inativação mais rápida. No terminal pré-sináptico, Lgi1 mutante falha em prevenir a rápida inativação do $\mathrm{K}_{\mathrm{v}} 1.1$, o resultado é a despolarização da membrana do neurônio e o influxo de íons $\mathrm{Ca}^{2+}$ através de canais sensíveis a voltagem. $\mathrm{O}$ grande influxo de $\mathrm{Ca}^{2+}$ provoca uma maciça liberação de vesículas contendo glutamato, gerando a hiperexcitabilidade. ${ }^{25}$

\section{$\mathrm{K}_{\mathrm{Ca}} 1.1$ (gene KCNMA1 - cromossomo 10q22.3)}

Canais de $\mathrm{K}^{+}$ativados pelo $\mathrm{Ca}^{2+}$ são tetrâmeros de subunidades $\alpha$ associadas a subunidades auxiliares $\beta^{26}$. Em pacientes portadores de uma síndrome que associa Epilepsia Generalizada e Discinesia Paroxística (GEPD) foi identificada uma mutação missense (D434G) no $\mathrm{K}_{\mathrm{Ca}} 1.1$, produto do gene KCNMA1. Essa mutação aumentou a sensibilidade do canal ao $\mathrm{Ca}^{2+}$, produzindo uma maior corrente de potássio. ${ }^{27}$

Yang et al. ${ }^{28}$ investigaram por qual mecanismo a mutação alterou a ativação sensível ao $\mathrm{Ca}^{2+}$ para resultar em ganho de função do canal. Para isso, utilizaram canais BK de ratos com a mutação D369G, que equivale a mutação D434G nos canais humanos. Tanto a mutação D369G quanto o sítio de ligação ao $\mathrm{Ca}^{2+}$, que é o resíduo D367, localizam-se dentro de uma região chamada AC no domínio RCK; e os resultados mostraram que a mutação provocou uma mudança dinâmica e conformacional nessa região, tornando-a mais rígida, o que levou os pesquisadores a conclusão de que uma região $\mathrm{AC}$ menos flexível pode ser mais efetiva na combinação da ligação ao $\mathrm{Ca}^{2+}$ para a ativação do canal.

\section{Canais de cálcio}

\section{Cav2. 1 (gene CACNA1A - cromossomo 19p13.2-p13.1)}

Alguns modelos de animais relacionam mutações na subunidade $\alpha_{1}$ dos $\mathrm{Ca}_{\mathrm{v}} 2.1$ com atividade epiléptica, mas em humanos poucas mutações foram identificadas, já que são principalmente associadas à Enxaqueca Hemiplégica Familiar e à Ataxia Episódica. ${ }^{29} \mathrm{O}$ primeiro caso familiar de epilepsia de ausência associada a uma mutação no CACNA1A foi identificado por Imbrici et al. ${ }^{30}$ Os pesquisadores encontraram a mutação missense E147K afetando o segmento S2 do DI e estudaram-na expressando-a, junto as subunidades $\alpha_{2} \delta 1$ e $\beta 4$, em oócitos de Xenopus. Os resultados eletrofisiológicos mostraram que a mutação E147K causou uma redução sutil na atividade do canal, em comparação com a mutação R1820X, mas os autores sugerem que esse comprometimento pode ter um papel central no desenvolvimento das crises.

\section{Ca 3.2 (gene CACNA1H - cromossomo 16p13.3)}

Variações genéticas no CACNA1H têm sido associadas à Epilepsia de Ausência da Infância (CAE), Epilepsia de Ausência Juvenil (JAE), Epilepsia Mioclônica Juvenil (JME) e Epilepsia Astática Mioclônica (MAE), mas não são suficientes para causar as síndromes. ${ }^{31}$ Mutações missense, anteriormente identificadas em pacientes com CAE, foram analisadas por Khosravani et al. ${ }^{32}$ utilizando células HEK293 transfectadas com canais $\mathrm{Ca}_{\mathrm{v}}$ 3.2. As mutações F161L e E282K resultaram em um desvio hiperpolarizante na ativação sensível à voltagem, enquanto que a V831M causou um desvio despolarizantes na inativação sensível à voltagem. Em neurônios, esse comportamento poderia gerar um maior influxo de $\mathrm{Ca}^{2+}$ pela abertura do canal em potenciais de membrana mais hiperpolarizados, resultando em oscilações do circuito tálamocortical e na propensão para as crises nos pacientes com CAE.

\section{Canal de cloreto}

\section{CIC-2 (gene CLCN2 - cromossomo 3q27. 1)}

Os canais ClC-2 são expressos em muitos tecidos. No cérebro, eles são encontrados nas células piramidais hipocampais e nas células da glia. ${ }^{33}$ São ativados pelo aumento do volume celular, pela acidifi- 
cação do pH extracelular e por hiperpolarização da membrana. Como os ClC-2 são expressos nos neurônios, é pensado que eles contribuem para o estabelecimento da baixa concentração intraneuronal de $\mathrm{Cl}^{-}$, um pré-requisito para a resposta inibitória do GABA. $\mathrm{O}$ gradiente eletroquímico de $\mathrm{Cl}^{-}$através da membrana plasmática determina se o GABA despolariza ou hiperpolariza a membrana pós-sináptica. ${ }^{34}$ De acordo com esse raciocínio, mutações que diminuam a atividade do $\mathrm{ClC}-2$ podem resultar em aumento da excitabilidade em certos neurônios.

Haug et al. ${ }^{35}$ identificaram três mutações no gene CLCN2 em três famílias com diferentes formas de epilepsia generalizada: um códon de terminação prematuro, uma mutação frameshift (que resultou em deleção de 44 aminoácidos) e uma mutação missense (G715E). Os pesquisadores estudaram as complicações funcionais das mutações utilizando células HEK293 transfectadas e os resultados mostraram que as duas primeiras mutações resultaram em perda de função do canal, que seria responsável pela diminuição do gradiente transmembrana de $\mathrm{Cl}^{-}$e comprometimento da inibição GABAérgica. Já a mutação G715E, surpreendentemente, aumentou a atividade do canal, levando à conclusão de que ambos, ganho e perda de função do ClC-2, podem causar epilepsia. Entretanto, mesmo o knockout do ClC-2 em ratos não resultou em epilepsia, fato que põe em dúvida as evidências de que as mutações no CLCN2 são epileptogênicas. ${ }^{36}$

\section{Subunidade $\alpha 1$ (gene GABRA1 - cromossomo 5q34-q35)}

Em indivíduos portadores de Epilepsia Mioclônica Juvenil (JME) de uma família canadense foi encontrada uma mutação missense (A322D) no gene GABRA1, que substituiu um resíduo de alanina por aspartato no segmento M3 da subunidade $\alpha 1$ do re-

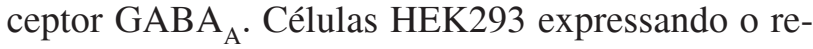
ceptor mutado $\left(\alpha 1_{\mathrm{A} 322 \mathrm{D}} \beta 2 \gamma 2\right)$ exibiram uma diminuição na corrente de $\mathrm{Cl}^{-}$, indicando que as crises podem surgir através da perda de função do receptor. ${ }^{37} \mathrm{Ou}-$ tros pesquisadores mostraram que a mesma mutação reduziu a expressão celular da subunidade $\alpha 1$ por causar sua rápida degradação associada ao retículo endoplasmático (ERAD) através do sistema ubiquitinaproteassoma ${ }^{38}$, e ainda diminuiu a expressão de receptores normais $(\alpha 1 \beta 2 \gamma 2$ e $\alpha 3 \beta 2 \gamma 2)$ pela associação da subunidade $\alpha 1_{\text {A322D }}$ com outras subunidades, prevenindo seu tráfego para a superfície. ${ }^{39}$

\section{Subunidade $\gamma 2$ (gene GABRG2 - cromossomo} 5q31.1-q33.1)

Uma mutação missense (R43Q) na subunidade $\gamma 2$ foi identificada em uma família com CAE e crises febris. ${ }^{40}$ Utilizando células HEK293 transfectadas, Bianchi et al. ${ }^{47}$ reportaram que a mutação R43Q diminuiu substancialmente a amplitude da corrente de $\mathrm{Cl}^{-}$, embora sem causar comprometimento na condutância ou no tempo de abertura do canal, sugerindo redução da expressão do receptor na superfície celular. Kang e Macdonald ${ }^{42}$ confirmaram que os receptores $\alpha 1 \beta 2 \gamma 2_{\mathrm{R} 43 \mathrm{Q}}$ tiveram sua expressão reduzida devido à retenção nos compartimentos intracelulares, incluindo o retículo endoplasmático. Essa redução resultaria em uma corrente inibitória GABAérgica diminuída in vivo e consequente aumento na excitabilidade neuronal e epilepsia.

\section{Subunidade $\beta 3$ (gene GABRB3 - cromossomo 15q11.2-q12)}

Tanaka et al. ${ }^{43}$ identificaram três mutações missense no gene GABRB3, que encoda a subunidade $\beta 3$ do receptor $\mathrm{GABA}_{\mathrm{A}}$, em pacientes com $\mathrm{CAE}$ oriundos da América Central. P11S, S15F e G32R acometem a porção $\mathrm{N}$-terminal extracelular do polipeptídio, um domínio importante para a ligação do GABA e de moduladores alostéricos. Ambas diminuíram a amplitude da corrente de $\mathrm{Cl}^{-}$e aumentaram a N-glicosilação do domínio extracelular, o que poderia afetar o processamento e posterior montagem dos receptores ${ }^{44}$. Os autores sugerem que o aumento na N-glicosilação afetaria a maturação e o tráfego dos receptores do retículo endoplasmático à membrana plasmática, resultando na redução da corrente do canal e surgimento das crises de ausência.

Em estudo mais recente, a mutação G32R foi responsável pelo aumento da expressão da subunidade $\beta 3$ e diminuição da expressão da subunidade $\gamma 2$, sugerindo uma substituição parcial de receptores $\alpha 1 \beta 3 \gamma 2$ por receptores $\alpha 1 \beta 3$ e homopentâmeros de subunidades $\beta 3$. Além disso, receptores $\alpha 1 \beta 3_{\mathrm{G} 32 \mathrm{R}} \gamma 2$ apresentaram um tempo médio de abertura mais curto, indicando que a mutação diminuiria a corrente do canal por alterar tanto a expressão dos receptores na superfície celular, quanto a cinética do canal. ${ }^{45}$

\section{Conclusão}

As drogas antiepilépticas (DAE) agem em canais iônicos para diminuir a excitabilidade neuronal, 
enquanto que as mutações, geralmente, resultam em um ganho de função nesses mesmos alvos. Isso prova a importância dos canais iônicos na epilepsia e suporta a constante busca por novos genes envolvidos no processo ictogênico. Devido à incapacidade de cura das DAE disponíveis atualmente, o grande desafio para as pesquisas científicas é a descoberta de um tratamento capaz de prevenir ou reverter a epileptogênese, beneficiando pessoas com risco conhecido devido a uma predisposição genética, histórico de lesão cerebral ou pacientes com curso progressivo de epilepsia.

\begin{abstract}
Data from the World Health Organization indicated a worldwide prevalence of epilepsy of about 50 million people. An epileptic seizure is a period of abnormal synchronous excitation of a neuronal population. The balance between control systems that prevent neurons from an excessive action potential discharge and other systems that facilitate excitation maintains the proper functioning of the central nervous system. This study aimed to collect information on role of channelopathies in the pathophysiological processess of epileptic seizures, along with considerations about antiepileptic therapy targeting ion channels. We performed a bibliographic review, using the databases Medline/PubMed, Scielo, Scirus, Wiley Online Library and Science Direct. The keywors used were: epilepsy, epileptic seizures, ion channels, channelopathies, antiepileptic drugs and epidemiology, in both Portuguese and English. Some epileptic syndromes were considered channelopathies after genetic investigations and therefore increased the understanding of the pathophysiology convulsive disorders. Given the inability to cure with currently available antiepileptic drugs, the major challenge for scientific research is the discovery of a treatment capable of prevent or reverse epilepsy.
\end{abstract}

Keywords: Epileptic Seizure. Ion Channels. Channelopathies.

\section{Referências}

1. Masia SL, Devinsky O. Epilepsy and behavior: a brief history. Epilepsy Behav. 2000;1:27-36.

2. World Health Organization (WHO). Fact sheet №999: Epilepsy. Disponível em:< http://www. who. int/mediacentre/ factsheets/fs999/en/index. html>. Acesso em: 23/05/2012.

3. Guilhoto LMFF, Muszkat RS, Yacubian EM. Consenso Terminológico da Associação Brasileira de Epilepsia. J Epilepsy Clin Neurophysiol. 2006;12:175-7.

4. Fisher RS, van Emde Boas W, Blume W, Elger C, Genton P, Lee $P$, et al. Epileptic seizures and epilepsy: definitions proposed by the International League Against Epilepsy (ILAE) and the International Bureau for Epilepsy. Epilepsia. 2005;46:470-2.

5. Scharfman HE. The neurobiology of epilepsy. Curr Neurol Neurosci Rep. 2007;7:348-54.

6. Steinlein OK. Genetic mechanisms that underlie epilepsy. Nat Rev Neurosci. 2004;5:400-8.

7. Cooper EC, Jan Ly. M-channels: neurological diseases, neuromodulation and drug development. Arch Neurol. 2003;60:496-500.

8. Gitaí DLG, Romcy-Pereira RN, Gitaí LLG, Leite JP, GarciaCairasco N, Paço-Larson ML. Genes e epilepsia I: epilepsia e alterações genéticas. Rev Assoc Med Bras. 2008;54:2728.

9. Weber $Y G$, Lerche $H$. Genetic mechanisms in idiopathic epilepsies. Dev Med Child Neurol. 2008;50:648-54.

10. Escayg A, Goldin AL. Sodium channel SCN1A and epilepsy: mutations and mechanisms. Epilepsia. 2010;51:1650-8.
11. Camfield P, Camfield C. Epileptic syndromes in childhood: clinical Features, outcomes, and treatment. Epilepsia. 2002;43(Suppl. 3):27-32.

12. Lossin C, Wang DW, Rhodes TH, Vanoye CG, George Jr AL. Molecular Basis of an Inherited Epilepsy. Neuron. 2002;34:877-84.

13. Meisler MH, Kearney JA. Sodium channel mutations in epilepsy and other neurological disorders. J Clin Invest. 2005;115:2010-17.

14. Engel Jr J. A proposed diagnostic scheme for people with epileptic seizures and with epilepsy: report of the ILAE task force on classification and terminology. Epilepsia. 2001;42:796-803.

15. Scalmani P, Rusconi R, Armatura E, Zara F, Avanzini G, Franceschetti $S$ et al. Effects in neocortical neurons of mutations of the Nav1.2 $\mathrm{Na}+$ channel causing Benign Familial Neonatal-Infantile Seizures. J Neurosci. 2006;26:10100-9.

16. Kamiya K, Kaneda M, Sugawara T, Mazaki E, Okamura N, Montal $\mathrm{M}$ et al. A nonsense mutation of the sodium channel gene SCN2A in a patient with intractable epilepsy and mental decline. J Neurosci. 2004;24:2690-8.

17. Holland KD, Kearney JA, Glauser TA, Buck G, Keddache M, Blankston $\mathrm{J}$ et al. Mutation of sodium channel SCN3A in a patient with cryptogenic pediatric partial epilepsy. Neurosci Lett. 2008;433:65-70.

18. Estacion M, Gasser A, Dib-Hajj SD, Waxman SG. A sodium channel mutation linked to epilepsy increases ramp and persistent current of Nav1.3 and induces hyperexcitability in hippocampal neurons. Exp Neurol. 2010;224:362-8. 
19. Meadows LS, Malhotra J, Loukas A, Thyagarajan V, KazenGillespie KA, Koopman MC et al. Functional and biochemical analysis of a sodium channel b1 subunit mutation responsible for Generalized Epilepsy with Febrile Seizures Plus Type 1. J Neurosci. 2002;22:10699-709.

20. Scheffer IE, Harkin LA, Grinton BE, Dibbens LM, Turner SJ, Zielinski MA et al. Temporal lobe epilepsy and GEFS+ phenotypes associated with SCN1B mutations. Brain. 2007;130:100-9.

21. Brown DA, Passmore GM. Neural KCNQ (Kv7) channels. Br J Pharmacol. 2009;156:1185-95.

22. Cooper EC, Aldape KD, Abosch A, Barbaro NM, Berger MS, Peacock WS et al. Colocalization and coassembly of two human brain M-type potassium channel subunits that are mutated in epilepsy. Proc Natl Acad Sci U. S. A. 2000;97:49149.

23. Staub E, Pérez-Tur J, Siebert R, Nobile C, Moschonas NK, Deloukas $P$ et al. The novel EPTP repeat defines a superfamily of proteins implicated in epileptic disorders. Trends Biochem Sci. 2002;27:441-4.

24. Schulte U, Thumfart JO, Klöcker N, Sailer CA, Bildl W, Biniossek $\mathrm{M}$ et al. The epilepsy-linked Lgi1 protein assembles into presynaptic Kv1 channels and inhibits inactivation by Kvb1. Neuron. 2006;49:697-706.

25. Caleo M. Epilepsy: synapses stuck in childhood. Nat Med. 2009;15:1126-7.

26. Latorre R, Brauchi S. Large conductance $\mathrm{Ca}^{2+}$-activated $\mathrm{K}^{+}$ (BK) channel: activation by $\mathrm{Ca} 2+$ and voltage. Biol Res. 2006;39:385-401.

27. Du W, Bautista JF, Yang H, Diez-Sampedro A, You SA, Wang $L$ et al. Calcium-sensitive potassium channelopathy in human epilepsy and paroxysmal movement disorder. Nat Genet. 2005;37:733-8.

28. Yang J, Krishnamoorthy G, Saxena A, Zhang G, Shi J, Yang , et al. An epilepsy/dyskinesia-associated mutation enhances BK channel activation by potentiating $\mathrm{Ca}^{2+}$ sensing. Neuron. 2010;66:871-83.

29. Pietrobon D. Cav2. 1 channelopathies. Pflugers Arch 2010;460(2):375-93

Pflugers arch., Eur. j. physiol.

30. Imbrici P, Jaffe SL, Eunson LH, Davies NP, Herd C, Robertson $R$ et al. Dysfunction of the brain calcium channel CaV2. 1 in absence epilepsy and episodic ataxia. Brain. 2004;127:268292.

31. Heron SE, Khosravani H, Varela D, Bladen C, Williams TC, Newman MR, et al. Extended spectrum of idiopathic generalized epilepsies associated with CACNA1 H functional variants. Ann Neurol. 2007;62:560-8.

32. Khosravani H, Altier C, Simms B, Hamming KS, Snutch TP, Mezeyova $\mathrm{J}$ et al. Gating effects of mutations in the Cav3.2 T-type calcium channel associated with Childhood Absence Epilepsy. J Biol Chem. 2004;279:9681-4.
33. Sík A, Smith RL, Freund TF. Distribution of chloride channel2-immunoreactive neuronal and astrocytic processes in the hippocampus. Neuroscience. 2000;101:51-65.

34. Staley K. The role of an inwardly rectifying chloride conductance in postsynaptic inhibition. J Neurophysiol. 1994;72:27384.

35. Haug K, Warnstedt M, Alekov AK, Sander T, Ramírez A, Poser $B$ et al. Mutations in CLCN2 encoding a voltage-gated chloride channel are associated with idiopathic generalized epilepsies. Nat Genet. 2003;33:527-32.

36. Blanz J, Schweizer M, Auberson M, Maier H, Muenscher A, Hübner $\mathrm{CA}$ et al. Leukoencephalopathy upon disruption of the chloride channel CIC-2. J Neurosci. 2007;27:6581-9.

37. Cossette $\mathrm{P}$, Liu L, Brisebois K, Dong H, Lortie A, Vanasse M et al. Mutation of GABRA1 in an autosomal dominant form of juvenile myoclonic epilepsy. Nat Genet. 2002;31:184-9.

38. Gallagher MJ, Ding L, Maheshwari A, Macdonald RL. The $\mathrm{GABA}_{A}$ receptor a1 subunit epilepsy mutation $\mathrm{A} 322 \mathrm{D}$ inhibits transmembrane helix formation and causes proteasomal degradation. Proc Natl Acad Sci U. S. A. 2007;104:12999-13004.

39. Ding L, Feng HJ, Macdonald RL, Botzolakis EJ, Hu N, Gallagher MJ. GABAA receptor a1 subunit mutation A322D associated with autosomal dominant juvenile myoclonic epilepsy reduces the expression and alters the composition of wild type GABAA receptors. J Biol Chem. 2010;285:26390405.

40. Wallace RH, Marini C, Petrou S, Harkin LA, Bowser DN, Panchal $R G$ et al. Mutant GABAA receptor ã2-subunit in childhood absence epilepsy and febrile seizures. Nat Genet. 2001;28:49-52.

41. Bianchi MT, Song L, Zhang $H$, Macdonald RL. Two different mechanisms of disinhibition produced by $\mathrm{GABA}_{\mathrm{A}}$ receptor mutations linked to epilepsy in humans. J Neurosci. 2002;22:5321-7.

42. Kang JQ, Macdonald RL. The GABAA receptor g2 subunit R43Q mutation linked to childhood absence epilepsy and febrile seizures causes retention of $\mathrm{a} 1 \mathrm{~b} 2 \mathrm{~g} 2 \mathrm{~S}$ receptors in the endoplasmic reticulum. J Neurosci. 2004;24:8672-7.

43. Tanaka M, Olsen RW, Medina MT, Schwartz E, Alonso ME, Duron RM et al. Hyperglycosylation and reduced GABA currents of mutated GABRB3 polypeptide in remitting childhood absence epilepsy. Am J Hum Genet. 2008;82:1249-61.

44. Buller AL, Hastings GA, Kirkness EF, Fraser CM. Site-directed mutagenesis of $\mathrm{N}$-linked glycosylation sites on the gammaaminobutyric acid type $A$ receptor alpha 1 subunit. Mol Pharmacol. 1994;46:858-65.

45. Gurba KN, Hernandez CC, Hu N, Macdonald RL. The GABRB3 mutation, G32R, associated with childhood absence epilepsy alters a1b3g2L $\mathrm{GABA}_{\mathrm{A}}$ receptor expression and channel gating. J Biol Chem. 2012;287:12083-97. 